\title{
Interference cancellation for non-orthogonal multiple access used in future wireless mobile networks
}

\author{
Xin $\mathrm{Su}^{1}$, HaiFeng $\mathrm{Yu}^{2}$, Wansoo $\mathrm{Kim}^{3}$, Chang $\mathrm{Choi}^{4}$ and Dongmin $\mathrm{Choi}^{5^{*}}$ (D)
}

\begin{abstract}
Non-orthogonal multiple access (NOMA) is suggested as a radio access candidate for future wireless mobile networks. It utilizes the power domain for user multiplexing on the transmitter side and adopts a successive interference cancellation (SIC) as the baseline receiver scheme, considering the expected mobile device evolution in the near future. However, recent research focuses more on the performance evaluation of NOMA in context of assuming the perfect $\mathrm{SIC}$ at receiver side. In order to clarify the performance gap between the perfect and the practical SIC in NOMA schemes, and to examine the possibility of applying NOMA with practical SIC, this paper investigates the performance of NOMA applying multi-input multi-output (MIMO) technology with zero-forcing (ZF) and minimum mean square error (MMSE) SIC schemes. We propose an analysis on error effects of the practical SIC schemes for NOMA and in addition propose an interference-predicted minimum mean square error (IPMMSE) IC by modifying the MMSE weight factor using interference signals. According to the IPMMSE IC and analysis of IC error effect, we further suggest the remaining interference-predicted MMSE (RIPMMSE) IC to cancel the remaining interference. The simulation results show that by considering practical IC schemes, the bit error rate (BER) is degraded compared with conventional orthogonal multiple access (OMA). This validates that the proposed IC schemes, which can predict the interference signals, provide better performance compared to NOMA with conventional ZF and MMSE IC schemes.
\end{abstract}

Keywords: Non-orthogonal multiple access, Interference cancellation, Minimum mean square error, Prediction

\section{Introduction}

In the fourth-generation (4G) mobile communication systems, such as Long-Term Evolution (LTE), WiMAX, LTEAdvanced, and V2V networks [1-3], orthogonal access based on orthogonal frequency division multiple access (OFDMA) or single carrier-frequency division multiple access (SC-FDMA) was adopted. Orthogonal access is a reasonable choice for achieving good system throughput with a simplified receiver design. However, due to the vastly increased need for high-volume services, such as image transfer, video streaming, and cloud-based services, a new mobile communications system with further enhancement of system throughput is required for the nextgeneration (5G) mobile communication systems. In order to fulfill such requirements, non-orthogonal multiple

\footnotetext{
* Correspondence: jdmcc@chosun.ac.kr

${ }^{5}$ Division of Undeclared Majors, Chosun University, Gwangju 61452, South Korea

Full list of author information is available at the end of the article
}

access (NOMA) with a successive interference cancellation (SIC) receiver in downlink was presented as one of several promising candidate radio access technologies [4-10]. For downlink NOMA, non-orthogonality is achieved by introducing the power domain, either in time/frequency/code domains, for user multiplexing. User de-multiplexing is obtained through the allocation of a large power difference between the users on the transmitter side and the application of SIC on the receiver side. In this case, everyone can use the overall transmission bandwidth to get higher spectrum efficiency, and better user fairness can be achieved, compared with conventional orthogonal multiple access (OMA), by assigning greater power to the users under poor channel conditions. Furthermore, NOMA is suitable for the situations of massive connectivity, because it can support more simultaneous connections.

NOMA is a candidate technology for further performance enhancements of LTE and LTE-Advanced, and both the concept and system performance are discussed 
and analyzed, considering the perfect SIC on the receiver side [11-16]. Therefore, in this paper, we exploit the system performance based on the link-level simulation (LLS) of NOMA with practical SIC schemes (i.e., zero-forcing [ZF] and MMSE SIC).

We analyzed the effect of the error that is caused by the interference cancellation (IC) by considering practical SIC schemes for NOMA in. Based on the analysis, we propose a novel interference-predicted minimum mean square error (IPMMSE) IC scheme for NOMA downlink, which is based on MMSE criteria from prediction about interference signals. Moreover, based on the IPMMSE IC and the analysis of the IC error effect, we propose the remaining interference-predicted MMSE (RIPMMSE) IC to cancel the remaining interference, which can further improve the system performance. The link-level evaluation is provided, and the simulation results show that by using the proposed IC schemes, the bit error rate (BER) performance is enhanced, compared with conventional IC schemes for NOMA.

The rest of the paper is organized as follows. Section 2 introduces the basics of NOMA and describes a NOMA system model with ZF and MMSE SIC schemes by using multi-input multi-output (MIMO) technology [17]. In Section 3, we compare the conventional multiple access (MA) and NOMA with the practical IC scheme and analyze the IC error effect for NOMA. Based on IC error analysis, the proposed IPMMSE IC and RIPMMSE IC schemes are described in Section 4. Finally, we conclude this paper in Section 5.

\section{System model}

\subsection{NOMA basics}

The basic NOMA scheme with SIC for a 2-user equipment (UE) case in the cellular downlink is illustrated in Fig. 1 [1]. The transmit information for $\mathrm{UE}_{i}(i=1,2)$ at the base station (BS) is $s_{i}$, with transmission power $p_{i}$, so the transmit signal for $\mathrm{UE}_{i}$ is

$$
x_{i}=\sqrt{p_{i}} s_{i}
$$

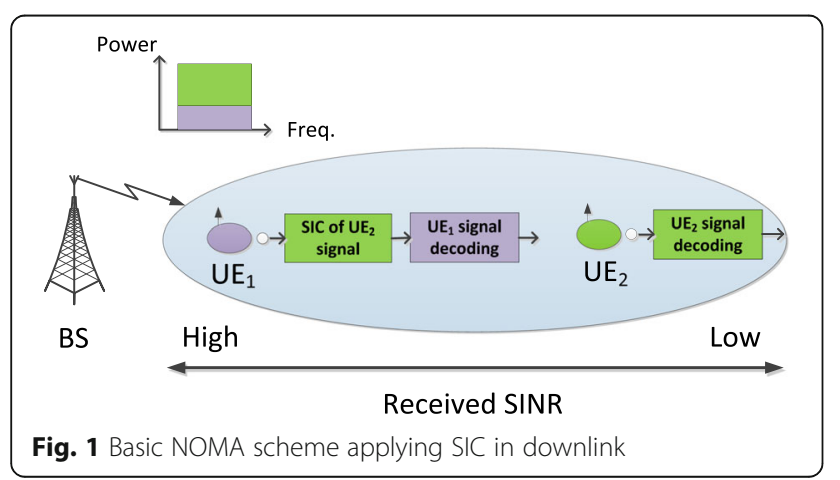

The sum of transmit power is restricted to $p$. So, the transmit signals are superposed as

$$
x=x_{1}+x_{2}=\sqrt{p_{1}} s_{1}+\sqrt{p_{2}} s_{2} .
$$

The received signal at $\mathrm{UE}_{i}$ is

$$
y_{i}=h_{i} x+n_{i}
$$

where $h_{i}$ is the complex channel coefficient between $\mathrm{UE}_{i}$ and the BS; $n_{i}$ denotes the receiver's Gaussian noise, including inter-cell interference. The power density of $n_{i}$ is $N_{0, i}$. In the NOMA downlink, decoding is in the order of the increasing channel gain normalized by the noise and inter-cell interference power, $\left|h_{i}\right|^{2} / N_{0, i}$. For a 2-UE case, as shown in Fig. 1, we assume that $\left|h_{1}\right|^{2} / N_{0,1}>\left|h_{2}\right|^{2} / N_{0,2}$, so $\mathrm{UE}_{1}$ first decodes $x_{2}$ and deletes its component from received signal $y_{1}$. And $\mathrm{UE}_{2}$ decodes $x_{2}$ without interference cancellation, because it has the first decoding order. The throughput of $\mathrm{UE}_{i}, R_{i}$ is

$$
R_{1}=\log _{2}\left(1+\frac{P_{1}\left|h_{1}\right|^{2}}{N_{0,1}}\right), \quad R_{2}=\log _{2}\left(1+\frac{P_{2}\left|h_{2}\right|^{2}}{P_{1}\left|h_{2}\right|^{2}+N_{0,2}}\right)
$$

\subsection{NOMA-MIMO with practical SIC schemes}

Sets of achievable rates for NOMA have been found by Cover [18], and the proof for the optimality of the sets of achievable rates for additive white Gaussian noise broadcast channels was given by Bergmans [19]. The capacity region of the uplink fading channel with receiver channel state information (CSI) was derived by Gallager [20], where he also showed that CDMA-type systems are inherently capable of higher rates than systems such as slow frequency hopping that maintain orthogonality between users. In [21], Tse gave a conclusion that NOMA is strictly better than OMA (except for the two corner points where only one user is being communicated to) in terms of sum rate, i.e., for any rate pair achieved by OMA, there is a power split for which NOMA can achieve rate pairs that are strictly larger. However, this conclusion is intended for single antenna systems and Tse did not give a proof for this conclusion. Here, one should be noted that the capacity gain of NOMA over OMA is achieved at the cost of more decoding complexity at the receivers for NOMA. The application of multiple-input multiple-output (MIMO) technologies to NOMA is important since the use of MIMO provides additional degrees of freedom for further performance improvement. The transceiver design for a special case of MIMO-NOMA downlink transmission, in which each user has a single antenna and the base station has multiple antennas, has been investigated in [22] and [23]. In [24], a multiple-antenna base station 
used the NOMA approach to serve two multiple-antenna users simultaneously, where the problem of throughput maximization was formulated and two algorithms were proposed to solve the optimization problem. In many practical scenarios, it is preferable to serve as many users as possible in order to reduce user latency and improve user fairness. Following this rationale, in [25], users were grouped into small-size clusters, where NOMA was implemented for the users within one cluster and MIMO detection was used to cancel inter-cluster interference. Different from conventional works, this paper focuses on the NOMA-MIMO with practical SIC instead of using perfect SIC assumption in conventional studies.

We assume orthogonal frequency division modulation (OFDM) signaling, although we consider non-orthogonal user multiplexing. Figure 2 illustrates the transmission scenario in the downlink NOMA-MIMO scheme for a 2$\mathrm{UE}$ case, where $\mathrm{UE}_{1}$ is a cell-center user (near user) and $\mathrm{UE}_{2}$ is a cell-edge user (far user). Figure 3 shows the block diagram of transmitter and receiver for the downlink NOMA scheme. We assume there are two transmission antennas at the BS, and each antenna transmits signal to one UE. The transmit signal $x_{i}$ for $\mathrm{UE}_{i}$ is

$$
x_{i}=\sqrt{p_{i}} s_{i},(i=1,2),
$$

where $p_{i}$ is the allocated power, and $s_{i}$ is the transmitted data for $\mathrm{UE}_{i}$. After transmitting through a $2 \times 2$ channel $\mathrm{H}$, as shown in Fig. 2, the 2-UE system is presented as

$$
Y=\left[\begin{array}{ll}
x_{1} & x_{2}
\end{array}\right]\left[\begin{array}{ll}
H_{11} & H_{12} \\
H_{21} & H_{22}
\end{array}\right]+\left[\begin{array}{ll}
n_{1} & n_{2}
\end{array}\right]
$$

where $n_{i}(i=1,2)$ is the receiver's Gaussian noise for $\mathrm{UE}_{i}$. The received signal $y_{i}$ for $\mathrm{UE}_{i}$ is represented as

$$
y_{i}=H_{i \mathrm{i}} x_{i}+\sum_{j \neq i} H_{j \mathrm{i}} x_{j}+n_{i},
$$

where $H_{j i}$ denotes the channel between the $j$ th antenna at the $\mathrm{BS}$ and the $i$ th receiver, $x_{j}$ is the transmit signal for $\mathrm{UE}_{j}$, which is the interference for $\mathrm{UE}_{i}$, and $n_{i}$ the Gaussian noise. After reception, the signals are ranked in decreasing order by power. Channel estimation (CE)

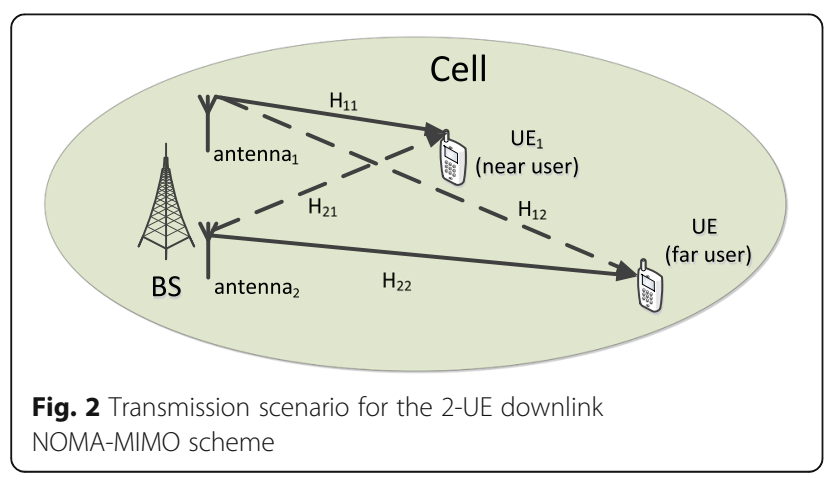

is performed for the interference signal $y_{j i}$ with power $p_{j}>$ $p_{i}$ (power of the desired signal). Then, SIC is employed until all interference signals are cancelled. The estimated received signal $\tilde{s}_{i}$ is obtained for $U E_{i}$. For the 2-UE case, from Eq. (6), the received signals for $\mathrm{UE}_{1}$ and $\mathrm{UE}_{2}$ are

$$
\begin{aligned}
& y_{1}=H_{11} x_{1}+H_{21} x_{2}+n_{1} \\
& y_{2}=H_{12} x_{1}+H_{22} x_{2}+n_{2}
\end{aligned},
$$

respectively. Since NOMA allows UEs to share the same resources, and differentiates UE by power, IC is performed for UE with lower power to cancel the inter-user interference. Because $\mathrm{UE}_{2}$ is a far user with greater power, the element $H_{21} x_{2}$ is cancelled by the IC from $y_{1}$, whereas $y_{2}$ can be demodulated directly without IC.

In this paper, we consider practical SIC schemes based on the zero-forcing (ZF) and the MMSE criteria. As described in the system model, by assuming power $p_{2} \gg p_{1}$, the $\mathrm{UE}_{2}$ can directly detect the signal without cancellation of interference. For $\mathrm{UE}_{1}$, the received signal $y_{1}$ is

$$
y_{1}=H_{11} \sqrt{p_{1}} s_{1}+H_{21} \sqrt{p_{2}} s_{2}+n_{1},
$$

where interference is $H_{21} \sqrt{p_{2}} s_{2}$. We can get the weight factor of channel $H_{21}$, i.e., $\left.\hat{H}_{21}\right|_{\mathrm{ZF}}$ or $\left.\hat{H}_{21}\right|_{\mathrm{MMSE}}$, by ZF or MMSE criteria after getting the estimated channel $\tilde{H}_{21}$ :

$$
\begin{aligned}
\left.\hat{H}_{21}\right|_{\mathrm{ZF}} & =\left(\tilde{H}_{21}^{H} \tilde{H}_{21}\right)^{-1} \tilde{H}_{21}^{H},\left.\hat{H}_{21}\right|_{\mathrm{MMSE}} \\
& =\left(\tilde{H}_{21}^{H} \tilde{H}_{21}+\sigma_{n_{1}}^{2} I\right)^{-1} \tilde{H}_{21}^{H},
\end{aligned}
$$

where the sign $H$ in the superscript in the equation represents the Hermitian transpose and $\sigma_{n_{1}}^{2}$ is the variance of noise, $n_{1}$. Then, the estimated interference signal can be obtained as

$$
\tilde{s}_{2} \approx{\sqrt{p_{2}}}^{-1} \hat{H}_{21} y_{1}=s_{2}+{\sqrt{p_{3}}}^{-1} \hat{H}_{21}\left(H_{11} \sqrt{p_{1}} s_{1}+n_{1}\right),
$$

The received signal is updated by subtracting the estimated interference signal:

$$
y_{1}{ }^{\prime}=y_{1}-\tilde{H}_{21} \sqrt{p_{2}} \tilde{s}_{2} \approx H_{11} \sqrt{p_{1}} s_{1}+n_{1} .
$$

After the canceling the interference signal with high power, $\mathrm{UE}_{1}$ can detect the desired information, $s_{1}$, from the updated received signal.

\section{IC error analysis}

3.1 Comparison between conventional MA and NOMA with practical IC scheme

In this section, we compare the packet error rate (PER) and throughput performances of conventional MA and NOMA by LLS. We still use the system model mentioned above for 2-UE. In conventional MA (without NOMA), the power for $\mathrm{UE}_{1}$ (near user), $p_{1}$, is assigned as 0.8 , and for $\mathrm{UE}_{2}$ (far user), $p_{2}$, is 0.2 , which follows the water filling (WF) algorithm. For NOMA, e.g., $p_{1}=0.2, p_{2}=0.8$ [4], 


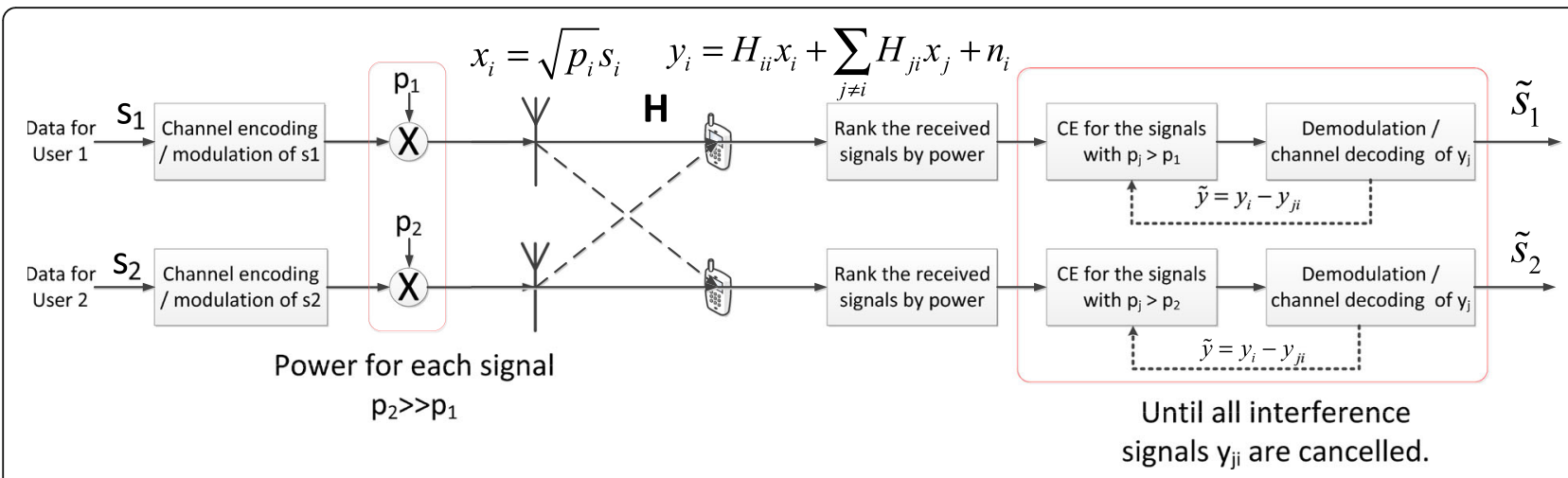

Fig. 3 Block diagram of transmitter and receiver for the downlink NOMA-MIMO scheme

which is power control (PC). We imitate system-level simulation (SLS) by LLS, where power allocation is used instead of path loss for cases without and with NOMA (i.e., WF versus PC). According to the LTE specifications, simulation parameters are summarized in Table 1. Figure 4 shows the PER performance for cases with NOMA (case_1) and without NOMA (case_2), where the practical ZF IC is applied for $\mathrm{UE}_{1}$ in case_2. According to the PER, the throughput $C$ can be calculated by

$$
C=\frac{(1-\mathrm{PER}) \times n_{\text {bit } / \text { packet }}}{T_{\text {packet }}(s)} .
$$

Therefore, the throughput performance for case_ 1 and case_2 is shown in Fig. 5. In the LLS, the path loss is not considered, and if we compare the performance between case_1 $\left(p_{1}=0.2, p_{2}=0.8\right)$ and case_2 $\left(p_{1}=0.8, p_{2}=0.2\right)$ under the scenario without NOMA, the performance of $\mathrm{UE}_{1}$ in case_1 is same as $\mathrm{UE}_{2}$ in case_2. And the performance of $\mathrm{UE}_{2}$ in case_1 is the same as $\mathrm{UE}_{1}$ in case_2. So, it is fair to compare the scenario of case_1 (with NOMA) and case_2 (without NOMA).

From the simulation results shown in Fig. 4, we determine that in NOMA, $\mathrm{UE}_{2}$ can detect information with

Table 1 Simulation parameters

\begin{tabular}{ll}
\hline Parameters & Value \\
\hline Bandwidth & $1.4 \mathrm{MHz}$ \\
Subcarriers per resource block & 12 \\
Symbols per packet & 6 \\
Resource blocks & 6 \\
$T_{\text {packet }}$ & $0.5 \mathrm{~ms}$ \\
Number of bits per packet $\left(n_{\text {bit/packet }}\right)$ & 864 \\
Channel & AWGN \\
Modulation & QPSK \\
Power for NOMA (case_1) & $p_{1}=0.2, p_{2}=0.8$ \\
Power for without NOMA (case_2) & $p_{1}=0.8, p_{2}=0.2$ \\
\hline
\end{tabular}

greater power than interference from $\mathrm{UE}_{1}$. Without NOMA, $\mathrm{UE}_{2}$ cannot detect information owing to the lower power compared to the interference signal from $\mathrm{UE}_{1}$. On the other hand, $\mathrm{UE}_{1}$ with NOMA can benefit from IC, even though the power of the interference signal is much higher than the target signal. Without NOMA, $\mathrm{UE}_{1}$ has performance similar to $\mathrm{UE}_{2}$ with NOMA, owing to the existing interference from the lower power user. As the results shown in Fig. 5, NOMA can increase the sum throughput and improve the fairness between the near and far users, compared with the situations without NOMA.

In order to evaluate the performance of NOMA under simulation, we provide comparisons of the LLS for $\mathrm{UE}_{1}$ under different modulation schemes, with the bound value for $\mathrm{UE}_{1}$ that is obtained from Eq. (4), mentioned above. Figure 6 shows the simulation curves for $U_{1}$ with NOMA under the perfect IC for different modulation and coding schemes. The coding scheme is convolutional code; the channel environment is single-path Rayleigh fading; and other simulation parameters are the same as in Table 1. From the comparison, all the simulation curves are lower than the bound and show a higher data rate with a higher order of modulation schemes. This verifies the LLS results and the analysis for the further research.

\section{IC error under AWGN channel}

For convenience, we use the same NOMA-MIMO transmission scenario as described in Section 2. At $\mathrm{UE}_{1}$ (near user), IC is applied to cancel the interference from the $\mathrm{UE}_{2}$ signal, as described in Fig. 7. Under the AWGN channel, the received signal for $\mathrm{UE}_{1}$ is

$$
y_{1}=x_{1}+x_{2}+n_{1},
$$

and owing to the larger power of $x_{2}$ compared to $x_{1}, x_{2}$ should be demodulated first. When demodulating $x_{2}$ at $\mathrm{UE}_{1}, x_{1}$ becomes the interference component: 


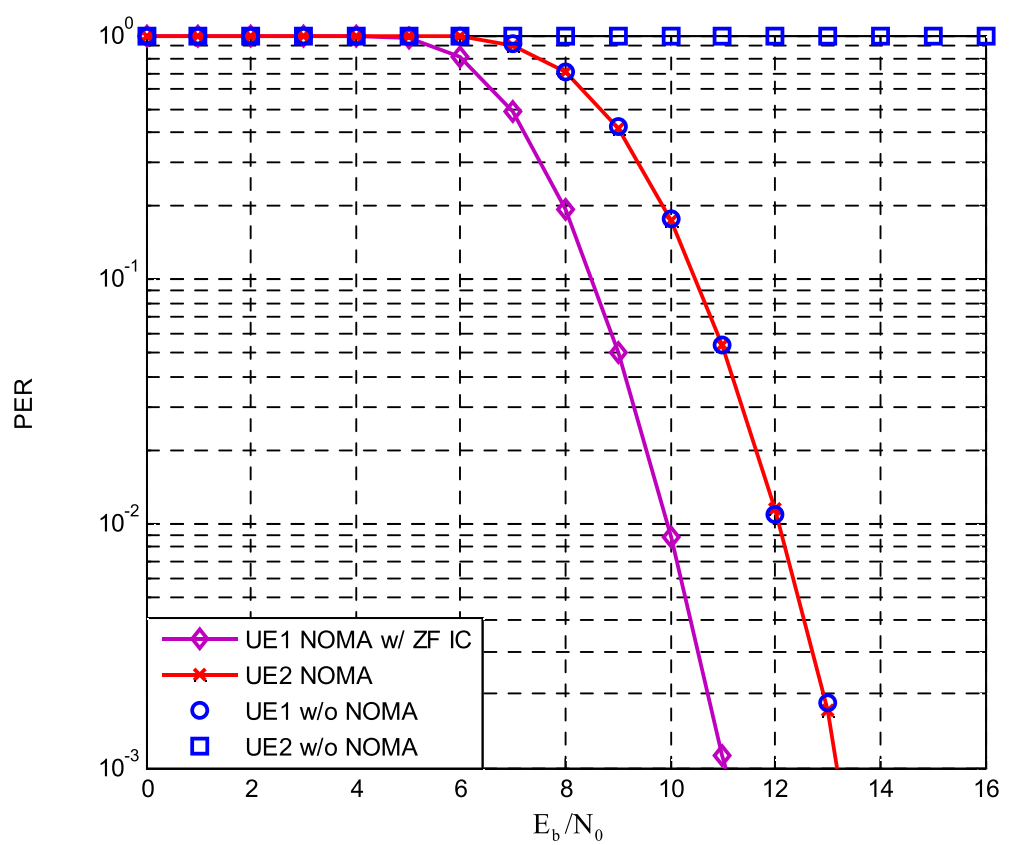

Fig. 4 PER performance with and without NOMA under AWGN channel

$$
y_{1}=x_{2}+\left(x_{1}+n_{1}\right)=x_{2}+i_{1 \_\mathrm{AWGN}}, \quad=\tilde{y}_{x_{2} \mid \mathrm{UE} 1}
$$

where $i_{1 \_ \text {AWGN }}$ is the interference for $x_{2}$ plus noise, and we assume that the demodulated signal of $x_{2}$ at $\mathrm{UE}_{1}$ is $\tilde{x}_{2 \mid \mathrm{UE} 1}$. Then, the noise enhancement ratio when demodulating $x_{2}$ at $\mathrm{UE}_{1}$ is

$$
e_{\mathrm{AWGN}}=\frac{i_{1 \_\mathrm{AWGN}}}{n_{1}} .
$$

After that, $\tilde{x}_{2 \mid \text { UE1 } 1}$ is re-modulated in order to cancel this signal from $y_{1}$, and the received signal after IC for $x_{2}$ is

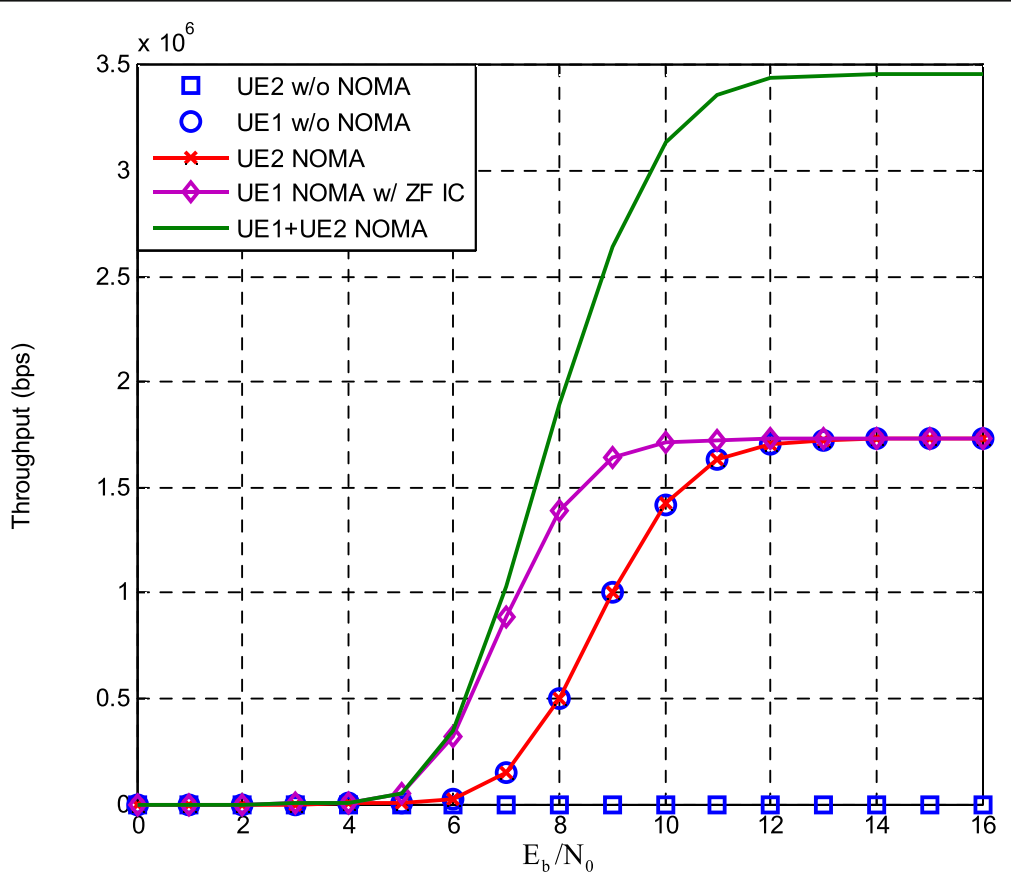

Fig. 5 Throughput performance with and without NOMA under AWGN channel 


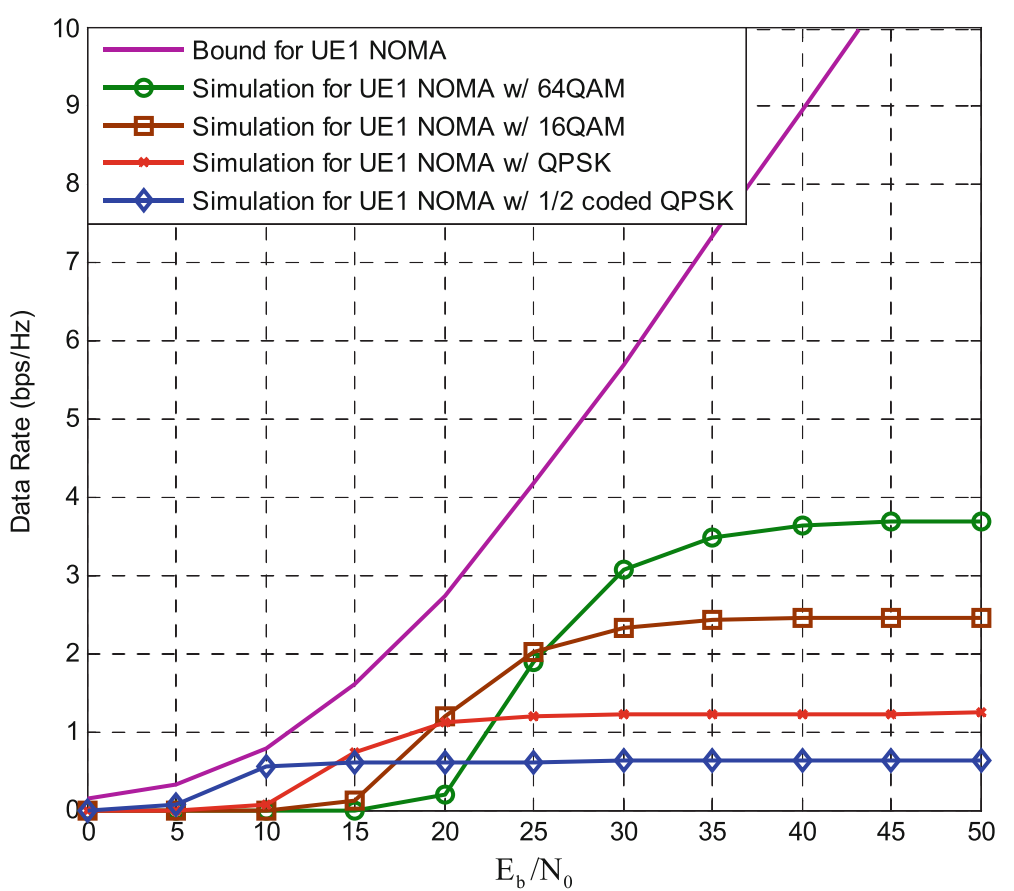

Fig. 6 Bound and simulation curves for $\mathrm{UE}_{1}$ under NOMA with different modulation schemes

$$
\begin{aligned}
y_{1}^{\prime} & =y_{1}-\hat{x}_{2} \\
& =x_{1}+\left(x_{2}-\hat{x}_{2}\right)+n_{1},
\end{aligned}
$$

where $\hat{x}_{2}$ is the re-modulated signal and the component $\left(x_{2}-\hat{x}_{2}\right)$ is the remaining interference for $x_{1}$ after IC. The power of the remaining interference for $x_{1}$ is

$$
p_{\left(x_{2}-\hat{x}_{2}\right)}=p_{2} \underbrace{\left(\frac{p_{2}}{i_{1 \_\mathrm{AWGN}}} / \frac{p_{2}}{n_{1}}\right)}_{\text {ratio of remaining interference }}=\frac{p_{2}}{e_{\mathrm{AWGN}}},
$$

where $\frac{p_{2}}{i_{1} \text { AWGN }}$ is the signal-to-interference-plus-noise ratio (SINR) and $\frac{p_{2}}{n_{1}}$ is the signal-to-noise ratio (SNR) of $x_{2}$ from perfect IC. Therefore, the bound value for $\mathrm{UE}_{1}$ after practical IC of $x_{2}$ is

$$
r_{1 \_C \_\mathrm{AWGN}}=\log _{2}\left(1+\frac{p_{1}}{\frac{p_{2}}{e_{\mathrm{AWGN}}}+n_{1}}\right) .
$$

Figure 8 shows the loss due to IC error for $\mathrm{UE}_{1}$ NOMA under practical IC compared with perfect IC (Fig. 6).

\subsection{ZF IC error under single-path Rayleigh channel}

Different from AWGN channel, various IC schemes can be used under the Rayleigh channel due to essentiality of channel equalization (CE). Firstly, we analyze ZF IC under the single-path Rayleigh channel. Similar to the AWGN case, the ZF IC is applied to cancel the interference from the $\mathrm{UE}_{2}$ signal, as described in Fig. 9. The received signal for $\mathrm{UE}_{1}$ is

$$
y_{1}=H_{11} x_{1}+H_{21} x_{2}+n_{1}
$$

where $H_{11}$ and $H_{21}$ are the channel coefficients for $x_{1}$ and $x_{2}$, respectively. Due to the larger power of $x_{2}, x_{2}$ should be demodulated first. When demodulating $x_{2}$ at $\mathrm{UE}_{1}, H_{11} x_{1}$ becomes the interference. After (perfect) ZF CE for $x_{2}$,

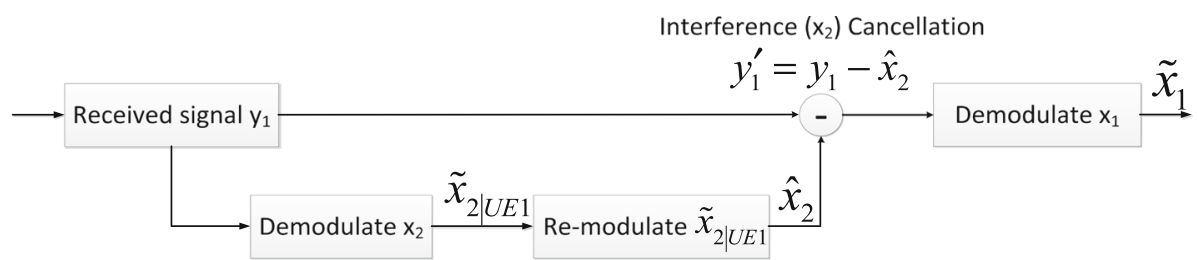

Fig. 7 Block diagram for IC under AWGN channel 


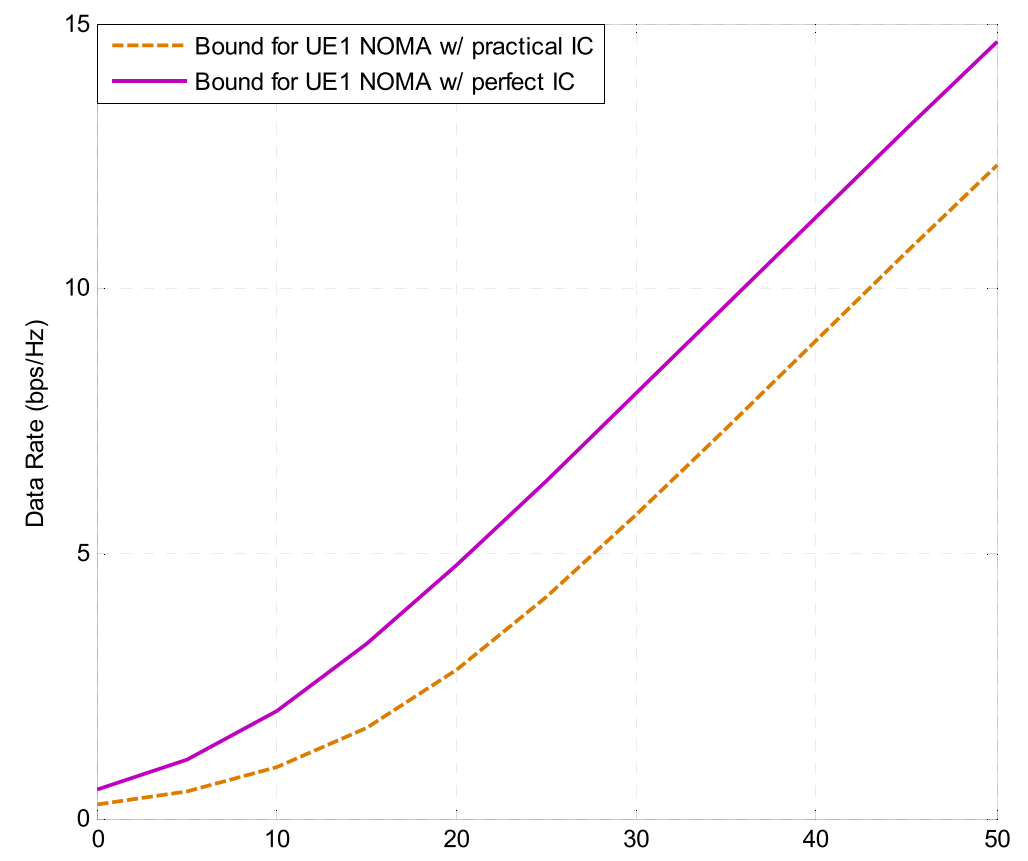

Fig. 8 Bound for $U E_{1}$ NOMA with perfect and practical IC under AWGN channel

$$
\begin{aligned}
H_{21}^{-1} y_{1} & =H_{21}^{-1} H_{11} x_{1}+x_{2}+H_{21}^{-1} n_{1} \\
& =x_{2}+H_{21}^{-1}\left(H_{11} x_{1}+n_{1}\right) \\
& =x_{2}+i_{1 \_Z F} \\
& =\tilde{y}_{x_{2} \mid \text { UE } 1}
\end{aligned}
$$

where $i_{1 \_ \text {ZF }}$ is the interference for $x_{2}$ plus noise, we assume the demodulated signal of $x_{2}$ at $\mathrm{UE}_{1}$ is $\tilde{x}_{2 \mid \mathrm{UE} 1}$. Then, the noise enhancement ratio when demodulating $x_{2}$ at $\mathrm{UE}_{1}$ is

$$
e_{\mathrm{ZF}}=\frac{i_{1 \_\mathrm{ZF}}}{n_{1}}
$$

After that, $\tilde{x}_{2 \mid \text { UE1 } 1}$ is re-modulated and then multiplied by the channel coefficient $H_{21}$ to cancel this signal from $y_{1}$. The received signal after ZF IC for $x_{2}$ is

$$
\begin{aligned}
y_{1}^{\prime} & =y_{1}-H_{21} \hat{x}_{2} \\
& =H_{11} x_{1}+H_{21}\left(x_{2}-\hat{x}_{2}\right)+n_{1},
\end{aligned}
$$

where $\hat{x}_{2}$ is the re-modulated signal and the component $H_{21}\left(x_{2}-\hat{x}_{2}\right)$ is the remaining interference for $x_{1}$ after ZF IC. The power of the remaining interference for $x_{1}$ is

$$
p_{\left(x_{2}-\hat{x}_{2}\right)}=p_{2} \underbrace{\left(\frac{p_{2}\left|H_{21}\right|^{2}}{i_{1 \_\mathrm{ZF}}} / \frac{p_{2}\left|H_{21}\right|^{2}}{n_{1}}\right)}_{\text {ratio of remaining interference }}=\frac{p_{2}\left|H_{21}\right|^{2}}{e_{\mathrm{ZF}}},
$$

where $\frac{p_{2}\left|H_{21}\right|^{2}}{i_{1 \_F F}}$ is the SINR and $\frac{p_{2}\left|H_{21}\right|^{2}}{n_{1}}$ is the SNR of $x_{2}$ from perfect IC. Therefore, the bound value for $\mathrm{UE}_{1}$ after ZF IC of $x_{2}$ is

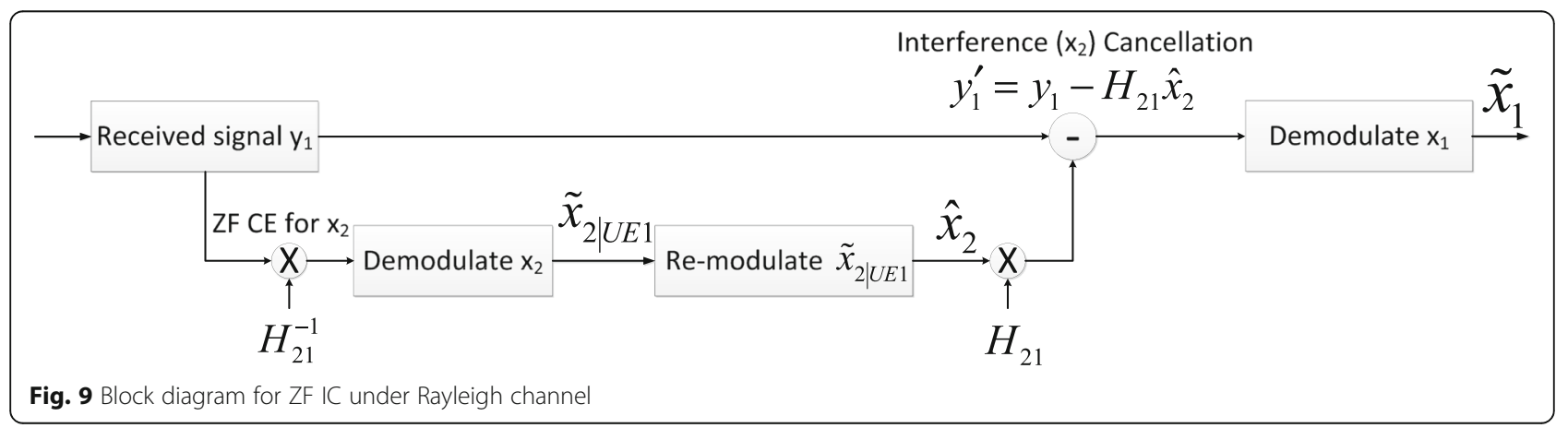




$$
r_{1 \_ \text {IC_ZF }}=\log _{2}\left(1+\frac{p_{1}\left|H_{11}\right|^{2}}{\frac{p_{2}\left|H_{21}\right|^{2}}{e_{\mathrm{ZF}}}+n_{1}}\right) .
$$

Figure 10 shows the bound and simulation curves for $\mathrm{UE}_{1}$ NOMA with perfect and practical ZF IC under the single-path Rayleigh channel. From the figure, both bound and simulation curves show the loss due to the practical IC error, compared with perfect IC.

\subsection{MMSE IC error under single-path Rayleigh channel}

Similar to ZF IC analysis, we analyze MMSE IC under the single-path Rayleigh channel. At $\mathrm{UE}_{1}$, the MMSE IC is applied to cancel the interference from the $\mathrm{UE}_{2}$ signal, as described in Fig. 11. The received signal for $\mathrm{UE}_{1}$ is

$$
y_{1}=H_{11} x_{1}+H_{21} x_{2}+n_{1}
$$

where $H_{11}$ and $H_{21}$ are the channel coefficients for $x_{1}$ and $x_{2}$, respectively. Due to the greater power of $x_{2}, x_{2}$ should be demodulated first. When demodulating $x_{2}$ at $\mathrm{UE}_{1}, H_{11} x_{1}$ becomes the interference. We assume perfect channel estimation, so the MMSE weight factor for channel $H_{21}$ is

$$
W_{\text {MMSE } \_H_{21}}=\left(H_{21}^{H} H_{21}+\sigma_{n_{1}}^{2} I\right)^{-1} H_{21}^{H},
$$

$$
\begin{aligned}
W_{\text {MMSE } \_H_{21}} y_{1} & =W_{\text {MMSE } \_H_{21}} H_{11} x_{1}+x_{2}+W_{\text {MMSE } \_H_{21}} n_{1} \\
& =x_{2}+W_{\text {MMSE } \_H_{21}}\left(H_{11} x_{1}+n_{1}\right) \\
& =x_{2}+i_{1 \_ \text {MMSE }} \\
& =\tilde{y}_{x_{2} \mid \text { UE } 1}
\end{aligned}
$$

where $i_{1 \_ \text {MMSE }}$ is the interference for $x_{2}$ plus noise, we assume the demodulated signal of $x_{2}$ at $\mathrm{UE}_{1}$ is $\tilde{x}_{2 \mid \mathrm{UE} 1}$. Then, the noise enhancement ratio when demodulating $x_{2}$ at $\mathrm{UE}_{1}$ is

$$
e_{\mathrm{MMSE}}=\frac{i_{1 \_\mathrm{MMSE}}}{n_{1}},
$$

After that, $\tilde{x}_{2 \mid \text { UE1 }}$ is re-modulated, then multiplied by the channel coefficient $H_{21}$ to cancel this signal from $y_{1}$. The received signal after MMSE IC for $x_{2}$ is

$$
\begin{aligned}
y_{1}^{\prime} & =y_{1}-H_{21} \hat{x}_{2} \\
& =H_{11} x_{1}+H_{21}\left(x_{2}-\hat{x}_{2}\right)+n_{1},
\end{aligned}
$$

where $\hat{x}_{2}$ is the re-modulated signal and the component $H_{21}\left(x_{2}-\hat{x}_{2}\right)$ is the remaining interference for $x_{1}$ after MMSE IC. The power of the remaining interference for $x_{1}$ is

$$
p_{\left(x_{2}-\hat{x}_{2}\right)}=p_{2} \underbrace{\left(\frac{p_{2}\left|H_{21}\right|^{2}}{i_{1 \_M M E}} / \frac{p_{2}\left|H_{21}\right|^{2}}{n_{1}}\right)}_{\text {ratio of remaining interference }}=\frac{p_{2}\left|H_{21}\right|^{2}}{e_{\mathrm{MMSE}}}
$$

After (perfect) MMSE CE for $x_{2}$

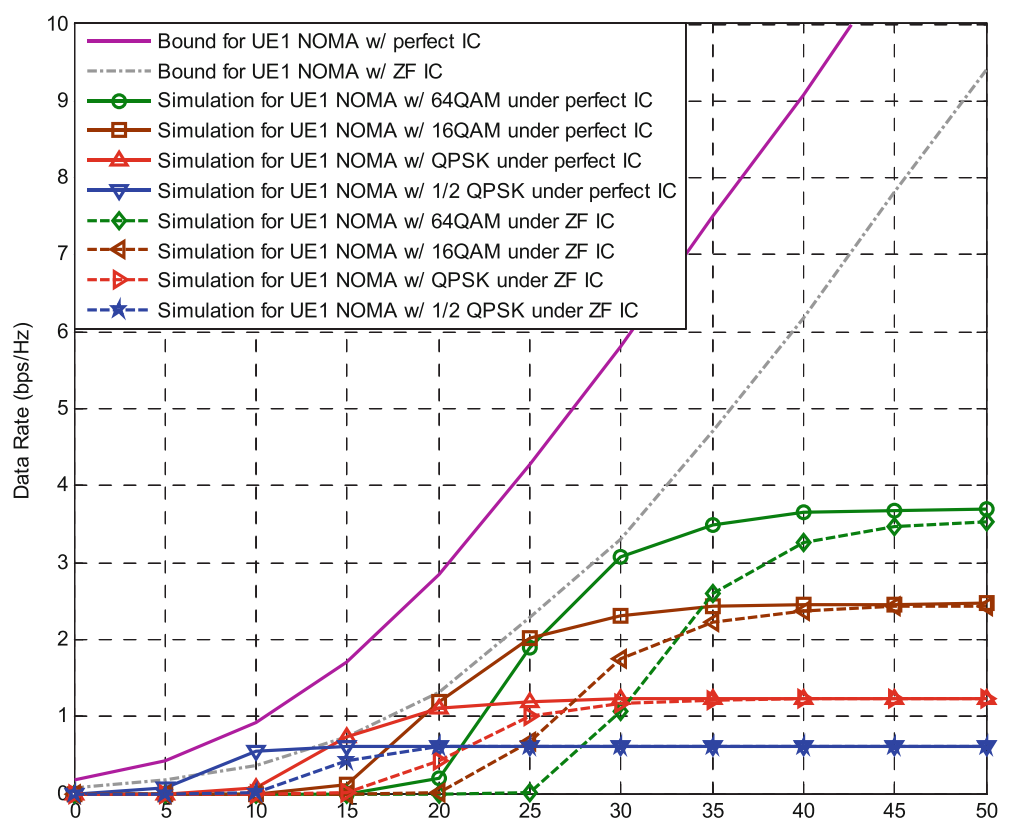

Fig. 10 Bound and simulation curves for $U E_{1}$ under NOMA with perfect and practical IC under single-path Rayleigh channel 


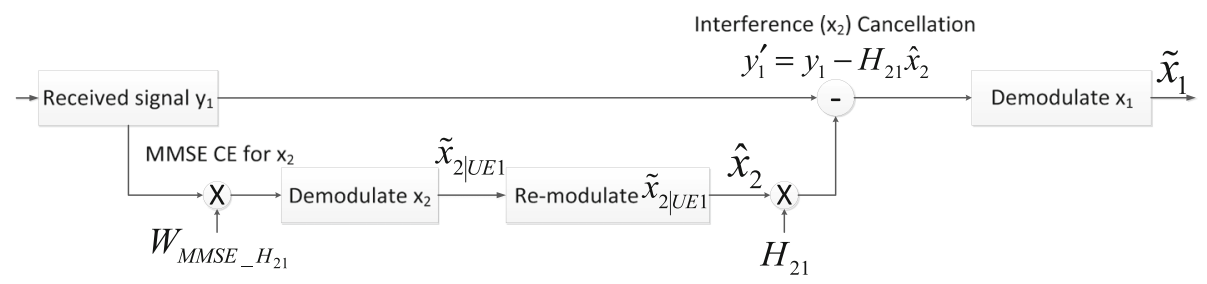

Fig. 11 Block diagram for MMSE IC under Rayleigh channel

where $\frac{p_{2}\left|H_{21}\right|^{2}}{i_{1} \text { MMSEE }}$ is the SINR and $\frac{p_{2}\left|H_{21}\right|^{2}}{n_{1}}$ is the SNR of $x_{2}$ from perfect IC. Therefore, the bound value for $\mathrm{UE}_{1}$ after MMSE IC of $x_{2}$ is

$$
r_{1 \_\mathrm{IC} \_\mathrm{MMSE}}=\log _{2}\left(1+\frac{p_{1}\left|H_{11}\right|^{2}}{\frac{p_{2}\left|H_{21}\right|^{2}}{e_{\mathrm{MMSE}}}+n_{1}}\right) \text {. }
$$

Figure 12 shows the bound and simulation curves for $\mathrm{UE}_{1}$ NOMA with practical ZF and MMSE IC under the single-path Rayleigh channel. From the simulation results, both bound and simulation curves show the gain from MMSE IC compared with ZF IC.

\section{Proposed interference-predicted MMSE IC schemes for NOMA}

5.1 Proposed interference-predicted MMSE IC scheme Based on the analysis of MMSE IC in the previous section, we propose modifying the MMSE weight factor by introducing the information on interference signals to improve the link-level performance of NOMA. From Eq. (28), we can determine that the interference for $x_{2}$ plus noise after MMSE CE for $x_{2}$ is

$$
i_{1 \_\mathrm{MMSE}}=W_{\text {MMSE_} H_{21}}\left(H_{11} x_{1}+n_{1}\right) .
$$

We assume that $i_{1}$ MMSE is the background noise for demodulating $x_{2}$, so then, the IPMMSE weight factor is

$$
W_{\text {IPMMSE_ } H_{21}}=\left(H_{21}^{H} H_{21}+\sigma_{i_{1} \_ \text {MMSE }}^{2} I\right)^{-1} H_{21}^{H},
$$

where $\sigma_{i_{1 \_\mathrm{MMSE}}}^{2}$ is obtained from the variance of $i^{1 \_\mathrm{MMSE}}$ :

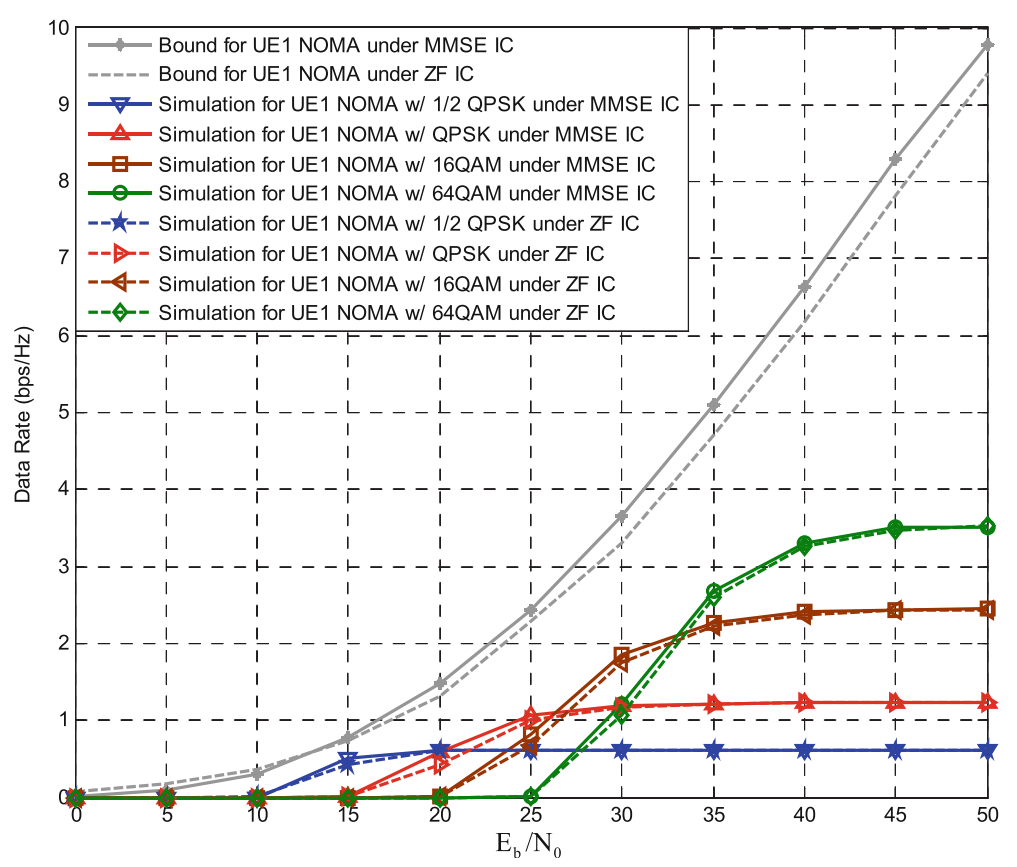

Fig. 12 Bound and simulation curves for $U E_{1}$ under NOMA with practical ZF and MMSE IC under single-path Rayleigh channel 


$$
\begin{aligned}
\operatorname{var}\left(i_{1 \_ \text {MMSE }}\right)= & E\left(i_{1 \_ \text {MMSE }} i_{1 \_ \text {MMSE }}^{H}\right) \\
= & E\left(\left(W_{\text {MMSE } \_H_{21}}\left(H_{11} x_{1}+n_{1}\right)\right)\left(W_{\text {MMSE } \_H_{21}}\left(H_{11} x_{1}+n_{1}\right)\right)^{H}\right) \\
= & W_{\text {MMSE } \_H_{21}} H_{11} E\left(x_{1} x_{1}^{H}\right) H_{11}^{H} W_{\text {MMSE } \_H_{21}}^{H} \\
& +W_{\text {MMSE } \_H_{21}} E\left(n_{1} n_{1}^{H}\right) W_{\text {MMSE } H_{21}}^{H} \\
= & W_{\text {MMSE_ } H_{21}} H_{11} \sigma_{x_{1}}^{2} H_{11}^{H} W_{\text {MMSE_ } H_{21}}^{H} \\
& +W_{\text {MMSE } \_H_{21}} \sigma_{n_{1}}^{2} W_{\text {MMSE } \_H_{21}}^{H}
\end{aligned}
$$

where the notation $E$ represents estimation. After deriving Eq. (35), we find that the MMSE weight factor can be modified by considering interference, which makes this algorithm practical. Equation (28) can be replaced by

$$
\begin{aligned}
W_{\text {IPMMSE } \_H_{21}} y_{1} & =W_{\text {IPMMSE } \_H_{21}} H_{11} x_{1}+x_{2}+W_{\text {IPMMSE } \_H_{21}} n_{1} \\
& =x_{2}+W_{\text {IPMMSE } \_H_{21}}\left(H_{11} x_{1}+n_{1}\right) \\
& =x_{2}+i_{1 \text { IPMMSE }} \\
& =\tilde{y}_{x_{2} \mid \text { UE } 1}
\end{aligned}
$$

Figure 13 shows the BER curves for $\mathrm{UE}_{1}$ with conventional OMA and NOMA for different IC schemes under single-path Rayleigh fading channel. The modulation and coding scheme used in the simulation is quadrature phase-shift keying (QPSK) with 1/2 convolutional code. From the simulation results, we find that OMA has the best $\mathrm{BER}$ performance, because there is no interference for OMA signaling. As for NOMA, ZF IC gives the worst BER, but it has the lowest complexity. And MMSE IC shows the better BER performance than ZF IC, which is in accordance with our previous analysis in Section 3. Finally, the proposed IPMMSE IC gives the best BER performance among the NOMA IC schemes, because it considers the effect of the interference signals. When the target BER is $10^{-3}$, the IPMMSE IC scheme outperforms the ZF IC scheme by around $1 \mathrm{~dB}$ and by $0.2 \mathrm{~dB}$ over the MMSE IC scheme.

\subsection{Proposed remaining interference-predicted MMSE IC scheme}

Based on the analysis of the IC error effect in the previous section, after IPMMSE CE, the noise enhancement ratio when demodulating $x_{2}$ at $\mathrm{UE}_{1}$ is

$$
e_{\mathrm{IPMMSE}}=\frac{i_{1 \_\mathrm{IPMMSE}}}{n_{1}}
$$

Then, the power of the remaining interference for $x_{1}$ is

$$
p_{H_{21}\left(x_{2}-\hat{x}_{2}\right) \_ \text {IPMMSE }}=p_{2}\left|H_{21}\right|^{2} \underbrace{\left(\frac{p_{2}\left|H_{21}\right|^{2}}{i_{1 \_ \text {IPMMSE }}} \mid \frac{p_{2}\left|H_{21}\right|^{2}}{n_{1}}\right)}_{\text {ratio of remaining interefreence }}=\frac{p_{2}\left|H_{21}\right|^{2}}{e_{\text {IPMMSE }}},
$$

where $\frac{p_{2}\left|H_{21}\right|^{2}}{i_{1 \perp \text { IPMMSE }}}$ is the SINR and $\frac{p_{2}\left|H_{21}\right|^{2}}{n_{1}}$ is the SNR of $x_{2}$ from perfect IC. The next step is to demodulate $x_{1}$, so we propose the remaining interference-predicted MMSE (RIPMMSE) IC for $x_{1}$ to cancel the remaining

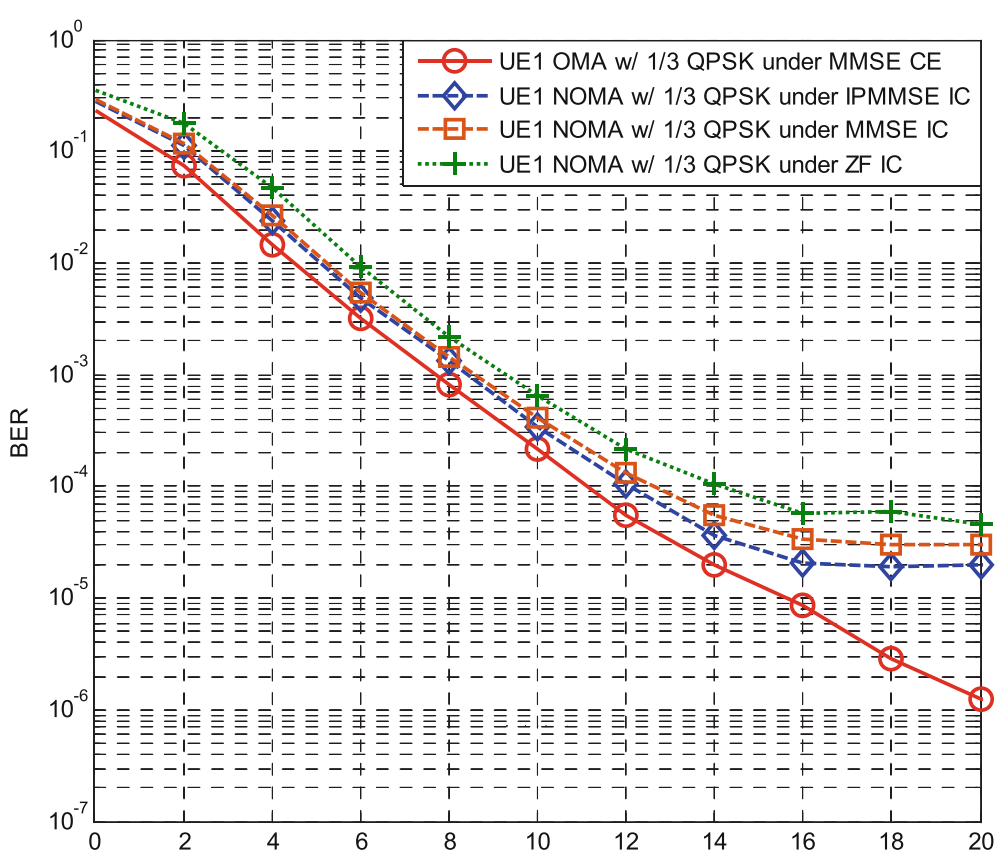

Fig. 13 BER for conventional OMA and NOMA with ZF, MMSE, and IPMMSE IC under single-path Rayleigh channel 
interference. The RIPMMSE weight factor for channel $H_{11}$ is

$$
W_{\text {RIPMMSE } \_H_{11}}=\left(H_{11}^{H} H_{11}+\sigma_{i_{1 \_ \text {IPMMSE }}}+n^{2} I\right)^{-1} H_{11}^{H},
$$

where $\sigma_{\left.i_{1}\right\lrcorner \text { PMMSE }}+n^{2}$ is obtained from the variance of the remaining interference plus noise

$$
\begin{aligned}
\operatorname{var}\left(H_{21}\left(x_{2}-\hat{x}_{2}\right)+n_{1}\right) & =E\left[\left(H_{21}\left(x_{2}-\hat{x}_{2}\right)+n_{1}\right)\left(H_{21}\left(x_{2}-\hat{x}_{2}\right)+n_{1}\right)^{H}\right] \\
& =E\left[\left(H_{21}\left(x_{2}-\hat{x}_{2}\right)+n_{1}\right)\left(\left(x_{2}-\hat{x}_{2}\right)^{H} H_{21}^{H}+n_{1}^{H}\right)\right] \\
& =E\left[H_{21}\left(x_{2}-\hat{x}_{2}\right)\left(x_{2}-\hat{x}_{2}\right)^{H} H_{21}^{H}\right]+E\left(n_{1} n_{1}^{H}\right) \\
& =H_{21} E\left[\left(x_{2}-\hat{x}_{2}\right)\left(x_{2}-\hat{x}_{2}\right)^{H}\right] H_{21}^{H}+\sigma_{n_{1}}^{2} \\
& =H_{21} p_{H_{21}\left(x_{2}-\hat{x}_{2}\right)_{\text {IPMMSE }}} H_{21}^{H}+\sigma_{n_{1}}^{2}
\end{aligned}
$$

After deriving Eq. (40), we find that the MMSE weight factor can be updated by considering the power of the remaining interference, which makes this algorithm practical. Figure 14 shows the BER performances for $\mathrm{UE}_{1}$ under conventional OMA and NOMA with different IC schemes under single-path Rayleigh fading channel. The modulation and coding scheme used in the simulation is QPSK with $1 / 3$ convolutional code. The simulation results show that RIPMMSE IC can further improve the BER performance compared to IPMMSE IC for NOMA. When the target BER is $10^{-3}$, the RIPMMSE IC scheme can provide a $1.5-\mathrm{dB}$ gain, compared to the ZF IC scheme, and a $0.5 \mathrm{~dB}$ gain over the MMSE IC scheme. Under the same principle, for 3-UE NOMA, the MMSE weight factor can be updated for a third time to further improve the BER performance.

\section{Conclusions}

In this paper, we exploit the performance of a NOMA-MIMO system for the perfect and the practical SIC schemes, which clarifies the necessity for the investigation into IC schemes for NOMA. Because many previous works focused on NOMA, some of the research topics, such as employment of the practical SIC and the error effect due to IC, are still in the early stages or not fully developed. In this paper, we perform the error analysis considering ZF and MMSE SIC schemes for NOMA; and based on the analysis, we propose a novel IPMMSE IC scheme by predicting the MMSE weight factor using information about interference signals. In addition, based on the IPMMSE IC scheme and the analysis of the IC error effect, we propose RIPMMSE IC to further boosts the system performance. We provide the link-level evaluations for a 2-UE scenario under NOMA-MIMO system on the single-path Rayleigh channel, and the simulation results show that the RIPMMSE IC scheme outperforms ZF and MMSE IC schemes by around 1.5 and $0.5 \mathrm{~dB}$, respectively, at a target BER of $10^{-3}$. In the future work, a more general case, i.e., larger number of users will be examined with our proposed schemes.

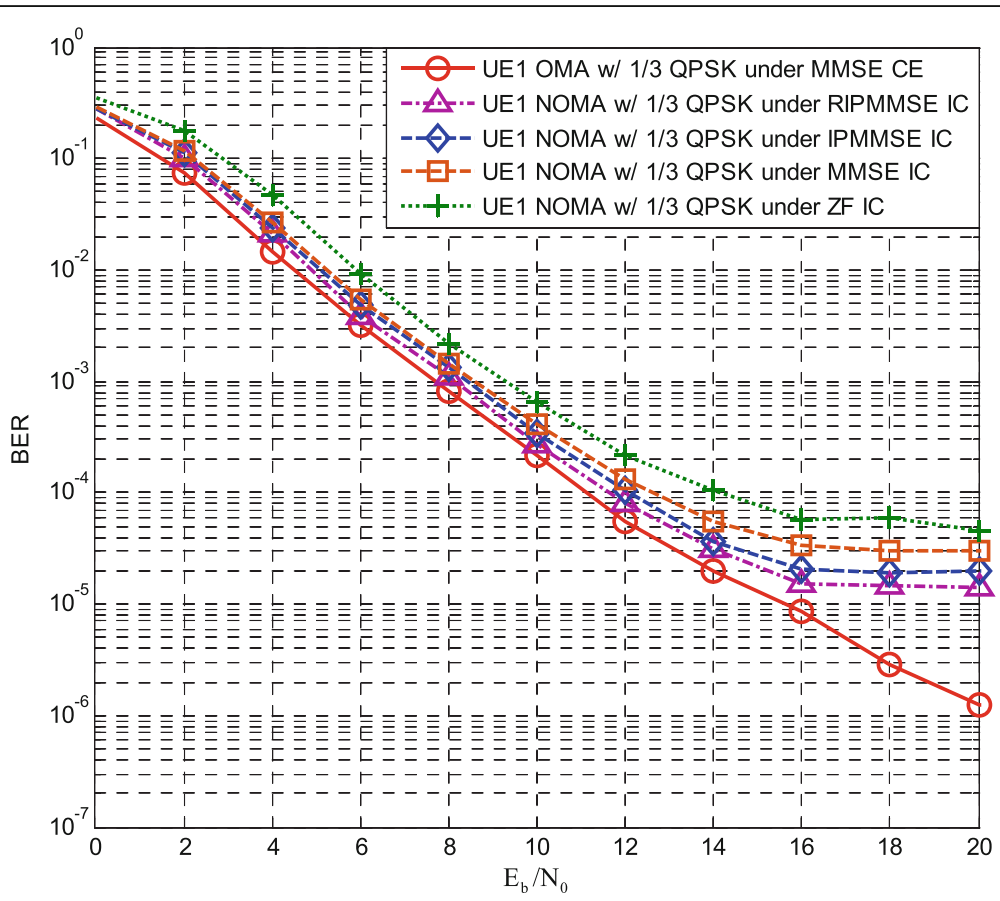

Fig. 14 BER for conventional OMA and NOMA with ZF, MMSE, IPMMSE, and RIPMMSE IC under single-path Rayleigh channel 


\section{Acknowledgements}

This work was supported in part by Fundamental Research Funds for the Central Universities under Grant 2015B30614, in part by the Natural Science Foundation of Jiangsu Province under Grant BK20160287, and in part by the National Research Foundation of Korea (NRF) grant funded by the Korea government (Ministry of Science, ICT and Future Planning) (No. NRF2015R1C1A1A01053301).

\section{Competing interests}

The authors declare that they have no competing interests.

\section{Author details}

'College of loT Engineering, Changzhou Key Laboratory of Robotics and Intelligent Technology, Hohai University, Changzhou, China. ${ }^{2}$ Carrier BG, Cloud Core Network Design and Bidding Department, Huawei Technologies CO., LTD, Shenzhen, China. ${ }^{3}$ Korea Institute for Curriculum and Evaluation, Seoul, South Korea. ${ }^{4}$ Department of Computer Engineering, Chosun University, Gwangju 61452, South Korea. 'Division of Undeclared Majors, Chosun University, Gwangju 61452, South Korea.

Received: 30 June 2016 Accepted: 15 September 2016

Published online: 27 September 2016

\section{References}

1. K Maxim, C Andrey, P Anton, K Igor, Technique of data visualization: example of network topology display for security monitoring. Journal of Wireless Mobile Networks, Ubiquitous Computing, and Dependable Applications (JoWUA) 7(1), 79-96 (2016)

2. B Bharat, AM Johnson, M Gisele Izera, A Pelin, A systematic approach for attack analysis and mitigation in V2V networks. Journal of Wireless Mobile Networks, Ubiquitous Computing, and Dependable Applications (JoWUA) 7(1), 97-117 (2016)

3. U Kim, J Kim, Research on object-oriented relational database model and its utilization for dynamic geo-spatial service through next generation ship navigation system, IT Convergence Practice 1(2), 1-10 (2013)

4. Y. Saito, Y. Kishiyama, A. Benjebbour, T. Nakamura, A. Li, and K. Higuchi, Non-orthogonal multiple access (NOMA) for cellular future radio access, in Proc. of IEEE VTC-Spring, 2013, pp. 1-5.

5. Umehara, and Y. Kishiyama, Enhancing user fairness in nonorthogonalaccess with successive interference cancellation for cellular downlink, in Proc. of IEEE ICCS, 2012, 324-328

6. K. Beomju, Non-orthogonal multiple access in a downlink multiuser beamforming system, in Proc. of IEEE MILCOM, 2013, 1278-1283.

7. A. Benjebbour, System-level performance of downlink NOMA for future LTE enhancements, in Proc. of IEEE GC Workshop, 2013, 66-70

8. L Dai, B Wang, Y Yuan, S Han, I Chih-Lin, Z Wang, Non-orthogonal multiple access for 5G: solutions, challenges, opportunities, and future research trends. IEEE Communications Magzine 53(9), 74-81 (2015)

9. B. Wang, K. Wang, Z. Lu, T. Xie, and J. Quan, Comparison study of nonorthogonal multiple access schemes for $5 \mathrm{G}$, in Proc. IEEE International Symposium on BMSB, 2015, 1-5

10. A Kitana, I Traore, I Woungang, Impact study of a mobile botnet over LTE networks. J Internet Serv Info Sec 6(2), 1-22 (2016)

11. N. Otao, Y. Kishiyama, and K. Higuchi, Performance of nonorthogonal access with SIC in cellular downlink using proportional fairbased resource allocation, in Proc. of IEEE ISWCS, 2012, 476-480

12. Q. Liu, B. Hui, and K.H. Chang, A survey on non-orthogonal multiple access schemes, in Proc. of KICS Winter Conf., 2014, 98-101.

13. A. Benjebbour, A. Li, Y. Saito, Y. Kishiyama, A. Harada, and T. Nakamura, System-level performance of downlink NOMA for future LTE enhancements, in Proc. of IEEE Globecom, 2013, 66-70

14. Saito, A. Benjebbour, Y. Kishiyama, and T. Nakamura, System-level performance of downlink non-orthogonal multiple access (noma) under various environments, in Proc. of IEEE VTC, 2015, 1-5

15. T. Seyama and H. Seki, Efficient selection of user sets for downlink nonorthogonal multiple access, in Proc. of IEEE PIMRC, 2015, 1062-1066

16. M.R. Hojeij, J. Farah, C.A. Nour, and C. Douillard, Resource allocation in downlink non-orthogonal multiple access (NOMA) for future radio access. in Proc. of IEEE VTC-Spring, 2015, 1-6
17. X Liu, H Miao, X Huang, A novel approach for blind estimation of a MIMO channel including phase unwrapping ambiguity elimination. IT Convergence Practice 1(2), 20-33 (2013)

18. TM Cover, Broadcast channels. IEEE Trans. Inf. Theory 18(1), 2-14 (1972)

19. PP Bergmans, A simple converse for broadcast channels with additive white Gaussian noise. IEEE Trans. Inf. Theory 20(2), 279-280 (1974)

20. RG Gallager, An inequality on the capacity region of multiaccess multipath channels, in Communications and Cryptography: Two Sides of One Tapestry (Kluwer, Boston, 1994), pp. 129-139

21. D. Tse and P. Viswanath, Fundamentals of wireless communication. Cambridge, Cambridge Univ. Press, 2005, ch. 6.

22. J Choi, Minimum power multicast beamforming with superposition coding for multiresolution broadcast and application to NOMA systems. IEEE Trans. Commun. 63(3), 791-800 (2015)

23. MF Hanif, Z Ding, T Ratnarajah, GK Karagiannidis, A minorizationmaximization method for optimizing sum rate in nonorthogonal multiple access systems. IEEE Trans. Signal Process 64(1), 76-88 (2006)

24. Q Sun, S Han, I C-L, Z Pan, On the ergodic capacity of MIMO NOMA systems. IEEE Wireless Commun. Lett. 4(4), 405-408 (2015)

25. Z Ding, F Adachi, HV Poor, The application of MIMO to nonorthogonal multiple access. IEEE Trans. Wireless Commun. 15(1), 537-552 (2016)

\section{Submit your manuscript to a SpringerOpen ${ }^{\circ}$ journal and benefit from:}

- Convenient online submission

- Rigorous peer review

- Immediate publication on acceptance

- Open access: articles freely available online

- High visibility within the field

- Retaining the copyright to your article

Submit your next manuscript at springeropen.com 